\title{
A Study of Unidirectionally Aligned Collagen-Silk Composite Fibers and the Application in hdpPSC Neural Differentiation
}

\author{
Bofan Zhu ${ }^{1}$, Wen $\mathrm{Li}^{1}$, Carlo Segre ${ }^{2}$, Randy Lewis ${ }^{3}$, Russ Janota ${ }^{4}$, Naiwei Chi ${ }^{1}$, Rong Wang ${ }^{1}$ \\ ${ }^{1 .}$ Department of Chemistry, Illinois Institute of Technology, Chicago, IL, 60616 \\ 2. Department of Physics, Illinois Institute of Technology, Chicago, IL, 606163 \\ 3 . Department of Biology, Utah State University, Logan, UT, 84322 \\ 4. Department of MMAE, Illinois Institute of Technology, Chicago, IL, 60616
}

Tissue engineering that involves matrix-specified differentiation of stem cells provides new approaches for tissue regeneration. In the preliminary study, collagen type I, as an important extracellular matrix (ECM) component, was found able to support cell proliferation and promote neuroectodermal commitment in stem cell differentiation [1] [2]. Other studies also showed aligned matrices can provide guidance for neural cell migration and directional axonal regeneration, which is a promising tissue engineering strategy for neural repair [3]. To create more suitable biological scaffolds, we used electrospinning technique to fabricate well-aligned collagen microfibers mimicking the native ECM. However, fibers made of pure collagen seemed to be too soft and unstable for long-term cell culture. Considering spider silk's remarkable tensile strength and superior elasticity, we incorporated silk proteins into collagen to enhance mechanical properties of e-spun fibers. In this study, we aim to reveal the role of unidirectionally aligned collagen-silk composite fibers in neural differentiation of human decidua parietalis placental stem cells (hdpPSCs).

Mechanical properties of the e-spun fibers were measured by stress-strain tests. Matrices with silk percentages (w/w) of 0\% (C), 15\% (CS15), 30\% (CS30), 60\% (CS60) and 100\% (S) were stretched along the fiber direction to generate stress-strain curves. As shown in Figure 1A, with the increase in the percentage of incorporated silk protein, the Young's modulus $(E)$ was found to increase from $0.581 \pm$ $0.048 \mathrm{GPa}$ for pure collagen to $4.452 \pm 0.658 \mathrm{GPa}$ for pure silk. The tensile strength also increased monotonously whereas the stretchability (strain) of fibers declined from $12.4 \%$ (C) to $5.4 \%$ (S). Thus, collagen-silk composites represent a compromise and are superior to either pure material.

Matrix stability, as another essential property for cell culture scaffolds, was evaluated by examining the change of fiber cross-sectional area and $E$ value in cell culture media using an AFM. By Day 7 of incubation, the cross-sectional area of collagen increased more than $100 \%$ with an $80.1 \%$ drop in fiber elasticity, suggesting an unstable matrix for long-term cell culture. On the other hand, the changes are much smaller in collagen/silk composites at an increased silk ratio (Fig. 1B). This result suggests that the incorporation of silk protein to collagen increased the matrix stability significantly.

To examine the effectiveness of the matrices to support stem cell differentiation, we grew hdpPSCs on the matrices. Cells were polarized by well-aligned fibers and developed long filaments along the fiber direction (Fig. 2A). However, fewer cells were observed on silk dominant fibers, suggesting a difference in cell adhesion among these fibers. In order to evaluate the cell-matrix adhesion strength, we carried out the immunostaining of integrin and a trypsin de-adhesion assay, and results showed that C, CS15 and CS30 matrices had higher integrin binding and stronger cell adhesion (Fig. 2B). The collagen-integrin interaction is known to stimulate neural differentiation of stem cells through a beta catenin pathway. We then examined the expression of two markers for in early stage of neural 
differentiation ( $\beta$-III-tubulin and Nestin) and found hdpPSCs had greater differentiation abilities towards neural cells when cultured on C, CS15 and CS30 e-spun matrices (Fig. 2C). This result was verified by immunostaining of NeuN - a maker for matured neural cells - at a later stage (data not shown). Taking the matrix stability into consideration, CS15 and CS30 composite fibers represent the favorable scaffolds for hdpPSCs neural differentiation.

On e-spun matrices, differentiated cells developed long neural filaments along the unidirectionally aligned fibers. Strikingly, we observed hdpPSCs experienced a much faster neural commitment (1 day vs. 3 days) when compared to those on random collagen gels [1]. The accelerated development to neural progenitors may arise from cell polarization that causes restricted integrin deposition along the fiber direction. The interplay of a mechanotransductive pathway may also facilitate the neural differentiation. To further investigate the collagen-integrin interaction, we will apply an AFM affinity measurement to quantify cell adhesion forces and to explore the distribution and potential structural changes of collagen within composite fibers. Taken together, collagen dominant composite fibers are mechanically strong, stable and provide excellent cell adhesion to promote stem cell differentiation. The aligned fibers can accelerate neural differentiation of stem cells, and promote 1D development of neural filaments which can be potentially utilized in future nano-bio-devices.

\section{References:}

[1] Indumathi Sridharan et al, BBRC, 437, (2013) 489-492

[2] Indumathi Sridharan, Taeyoung Kim, and Rong Wang, BBRC, 381, (2009) 508-512

[3] Yee-Shuan Lee, Treena Livingston Arinzeh, Polymers. 3, (2011) 422-423.

(A) Mechanical property of electrospun fibers

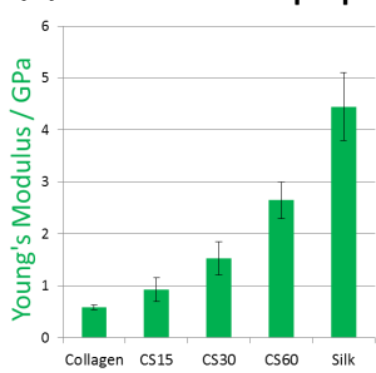

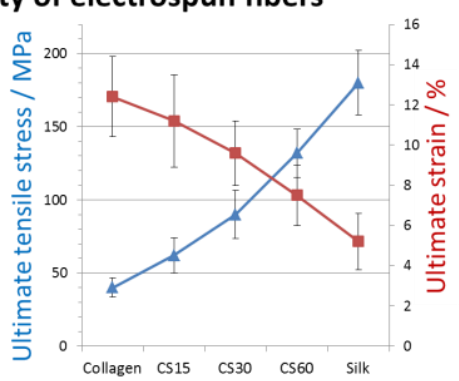

(B) AFM measurement of fiber cross-sectional area

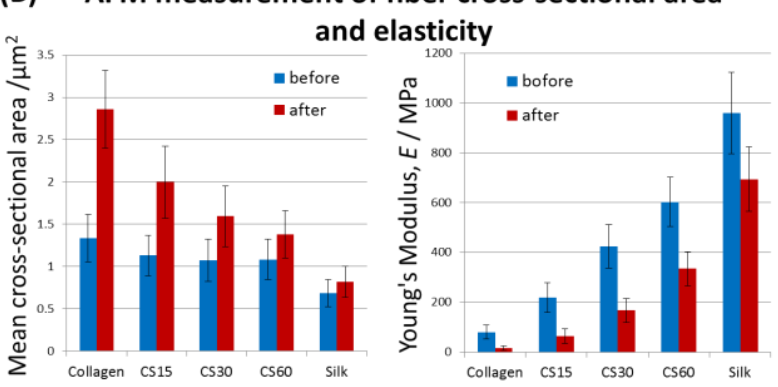

Figure 1. (A) Young's modulus, ultimate tensile strength and ultimate strain of e-spun matrices. (B) AFM measurement of fiber cross-sectional area and elasticity before and after 7 days incubation.

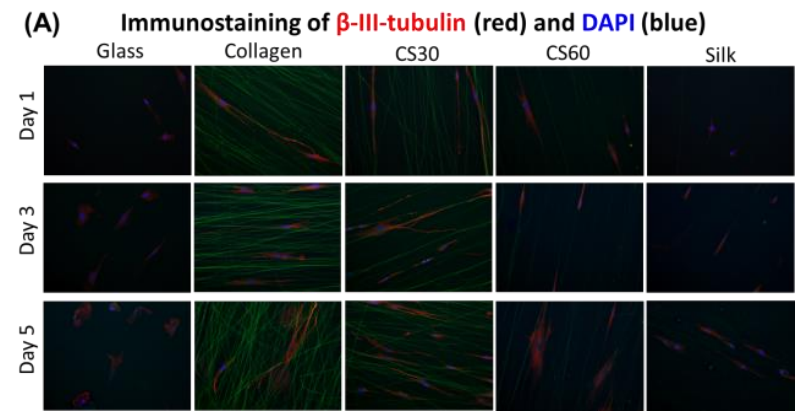

(B) Cell-matrix adhesion

(C) Neural marker expression
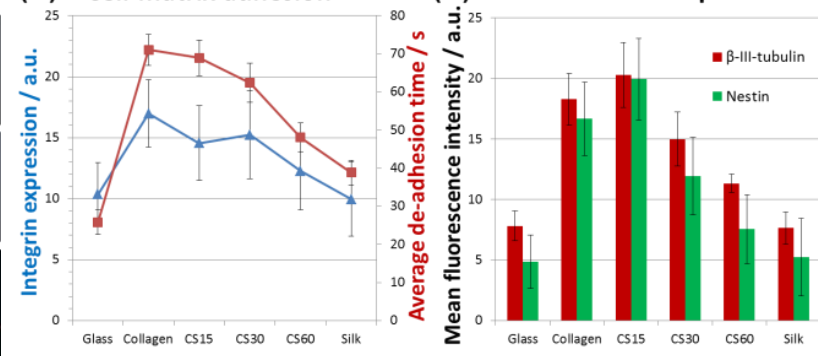

Figure 2. (A) Immunofluorescent images showing $\beta$-III-tubulin (red) positive cells on various matrices at Day 1, 3 and 5 of differentiation. (B) Cell adhesion characterized by mean fluorescence intensity of $\beta-1$ integrin (blue) and cell de-adhesion time (red). (C) ImageJ quantification of the expression levels of $\beta$-III-tubulin (red) and Nestin (green) at Day 1. 